\title{
Ligny-le-Châtel (Yonne) : fouille archéologique partielle d'une nécropole mérovingienne et d'un village déserté
}

Patrick Chopelain, Sandrine Thiol et Marie-Agnès Widehen

\section{(2) OpenEdition \\ 1 Journals \\ Édition électronique \\ URL : https://journals.openedition.org/cem/172 \\ DOI : $10.4000 /$ cem. 172 \\ ISSN : 1954-3093 \\ Éditeur \\ Centre d'études médiévales Saint-Germain d'Auxerre}

\section{Édition imprimée}

Date de publication : 15 août 2006

ISSN : 1623-5770

Référence électronique

Patrick Chopelain, Sandrine Thiol et Marie-Agnès Widehen, « Ligny-le-Châtel (Yonne) : fouille archéologique partielle d'une nécropole mérovingienne et d'un village déserté », Bulletin du centre d'études médiévales d'Auxerre | BUCEMA [En ligne], 10 | 2006, mis en ligne le 15 septembre 2006, consulté le 22 septembre 2022. URL : http://journals.openedition.org/cem/172 ; DOI : https://doi.org/ 10.4000/cem. 172

Ce document a été généré automatiquement le 22 septembre 2022.

\section{c) (7) (2)}

Creative Commons - Attribution - Pas d'Utilisation Commerciale - Partage dans les Mêmes Conditions 4.0 International - CC BY-NC-SA 4.0

https://creativecommons.org/licenses/by-nc-sa/4.0/ 


\title{
Ligny-le-Châtel (Yonne) : fouille archéologique partielle d'une nécropole mérovingienne et d'un village déserté
}

\author{
Patrick Chopelain, Sandrine Thiol et Marie-Agnès Widehen
}

1 L'intervention archéologique ${ }^{1}$ qui s'est déroulée au lieu-dit " La Coupe au Meunier » durant le printemps et l'automne 2005 est liée à un projet de déviation routière ${ }^{2}$ permettant de délester le bourg de Ligny-le-Châtel, chef-lieu de canton situé dans la vallée du Serein entre Chablis et Pontigny. Un diagnostic réalisé par l'INRAP en 2002 a révélé la présence de sarcophages mérovingiens, de structures d'habitat protohistoriques et médiévales ${ }^{3}$. Cet ensemble de parcelles, à quelques centaines de mètres au sud de l'agglomération actuelle correspond au site d'un habitat médiéval déserté connu sous le nom de "Ligny-la-Ville», qui serait également à proximité d'un monastère carolingien dédié à Saint-Symphorien ${ }^{4}$.

La nécropole mérovingienne (et carolingienne?)

2 La fouille a porté sur 166 tombes sur un total d'un peu plus de 200 répertoriées (un nombre important de structures non fouillées s'engageaient au-delà des limites de fouille).

3 Le décapage effectué sur l'emprise de la fouille n'a livré que les limites nord et est d'un ensemble beaucoup plus important. Par ailleurs, si l'orientation des tombes est le plus couramment ouest-est, nous avons pu différencier une trentaine de tombes orientées sud-ouest/nord-est sur le pourtour de la zone de plus forte concentration, indiquant une phase d'inhumation postérieure. Celles-ci sont axées sur un fossé dont le comblement a révélé un mobilier céramique de la Tène finale. Cependant, la présence dans plusieurs cas de pierres de calage, vraisemblablement issues de fragments de sarcophages récupérés, et l'absence de mobilier nous incitent à dater ces tombes de la période carolingienne. Des analyses radiocarbone sont en cours. 
4 La partie mérovingienne de la nécropole se présente comme un espace compact et dense à l'intérieur duquel il est toutefois possible de dégager quelques caractéristiques : la bordure nord est très régulière, longue d'une cinquantaine de mètres; la limite sud présente une organisation confuse.

5 L'orientation ouest-est est commune à la plupart des structures. À l'intérieur de ce périmètre, le rassemblement des sarcophages par deux ou trois, ou alors par six, disposés de façon rayonnante, indiqueraient vraisemblablement l'appartenance des inhumés à un même groupe, familial ou autre.

6 Le mobilier, recueilli dans 47 sépultures, montre que la nécropole fut utilisée $\mathrm{du} \mathrm{VI}^{\mathrm{e}}$ au $\mathrm{VII}^{\mathrm{e}}$ siècle ${ }^{5}$. Excepté quelques perles, l'essentiel du matériel est en métal. Il concerne avant tout des garnitures de ceinture et des bagues. À chaque fois que l'observation était permise par la conservation des tombes, le défunt portait ces objets personnels. Quelques tombes datées de la deuxième moitié du viI ${ }^{\mathrm{e}}$ siècle se retrouvent en périphérie $\mathrm{du}$ cimetière, non loin de tombes plus anciennes. Nous comptons très peu de mobilier associé, et la logique d'implantation et de développement de cette nécropole est encore en phase d'étude. Le faciès culturel perçu à travers le mobilier correspond bien à l'emplacement géographique de la nécropole (marges du Bassin Parisien et proximité de la France orientale) ${ }^{6}$. Une cruche à pied balustre et panse ovoïde constituant l'unique dépôt funéraire, est d'un type connu en Bourgogne du Nord et en Champagne ${ }^{7}$ tandis qu'elle se rencontre rarement en Bourgogne orientale et méridionale. Trois plaques damasquinées de la première moitié $\mathrm{du}_{\mathrm{vII}}^{\mathrm{e}}$ siècle à cinq ou six bossettes, ainsi qu'un exemplaire de type aquitain nous renvoie à une forme de mobilier témoignant de la proximité de l'île-de-France et de la moyenne vallée de la Seine. Une plaque-boucle à queue d'aronde, proche du type A évoque plutôt la France du Centre-Est. Parmi le matériel précoce, les éléments de baudrier d'épée de la tombe 98 (boucle simple, plaque carrée et applique) seraient peut-être l'indice du rang social élevé du défunt, hypothèse toutefois à nuancer par la présence également dans la tombe d'une garniture de ceinture dépareillée (plaque-boucle en alliage cuivreux, ardillon et plaque dorsale en fer).

7 Si on considère, la plupart du temps, que les couvercles de sarcophage structuraient le paysage funéraire, il n'en est rien à Ligny puisque la trace de petits dômes de graviers a été repérée au-dessus de certains d'entre eux. On ne peut être affirmatif pour chacun d'entre eux, dans la mesure où, un certain nombre de couvercles de sarcophages ont été «accrochés» par le soc de charrue. Une trace de marquage au sol, de matérialisation en surface, est à signaler; elle concerne un cas de superposition exacte de deux tombes.

8 Parmi ces 166 tombes, 60 sarcophages abritant de un à trois individus ont été répertoriés, les autres étant constituées de contenants périssables. Les sarcophages semblent souvent légèrement trop étroits tant en longueur qu'en largeur, comme s'il existait une production standardisée de ces derniers, négligeant la morphologie de l'individu. L'étude taphonomique a permis de restituer parmi les architectures périssables dix-sept cercueils ou coffrages chevillés larges, trente cercueils ou coffrages chevillés ajustés, onze cercueils ou coffrages chevillés étroits, les données ne permettant pas toujours de distinguer entre cercueil ou coffrage. Huit structures coffrées et surmontées ou non d'une couverture de planches pourraient avoir accueilli un individu dans une enveloppe souple. Enfin, quatre structures semblent avoir bénéficié d'une couverture de planches et six tombes ont été considérées comme des 
inhumations en pleine terre de sujets munis d'une enveloppe funéraire. Huit structures se présentent sous la forme de fosses sépulcrales (mêmes dimensions, même orientation) mais elles accueillent des lots d'os déconnectés, regroupés sur l'un ou l'autre des bords de la structure. Il pourrait s'agir de tombes curées dans la perspective de l'arrivée d'un nouveau cadavre et qui, finalement, n'auraient pas été réutilisées. Le mauvais état de conservation de huit structures n'a pas permis l'identification du type d'architecture initial.

9 L'étude biologique des individus est en cours. Pour autant, la collection semble d'ores et déjà particulièrement intéressante sur le plan paléo-pathologique avec de nombreux foyers d'appositions périostées renvoyant à des infections. En outre, une trace de violence, n'ayant pas entraîné une mort immédiate, a été observée sur le dessus du crâne d'un sujet adulte. La blessure s'est consolidée sans prolifération osseuse anarchique indiquant une réparation sans infection. Le phénomène semble isolé dans la « collection » et nous pensons qu'il résulte d'un épisode de violence ponctuel.

Les regroupements de sarcophages permettaient de supposer l'existence de liens de parenté entre les individus ainsi associés sur le terrain. Afin de vérifier cette hypothèse, des prélèvements (un os de chaque pied) ont été effectués chaque fois que la conservation du squelette le permettait. Des mesures ont été prises sur le terrain afin d'éviter tout contact direct avec l'échantillon prélevé et donc toute contamination de l'ADN ancien par de l'ADN contemporain (charlotte, protège nez/bouche, combinaison jetables, gants et scalpels stériles). 223 prélèvements ADN ont été effectués dans 114 structures soit sur un peu moins de $70 \%$ des tombes. L'analyse de l'ensemble des échantillons permettra, entre autres, de mettre en relation ou pas les rassemblements de sarcophages sur le terrain avec les liens de parenté possibles entre les individus inhumés.

L'habitat du bas Moyen Âge et le problème des villages-doublons : Ligny, un cas d'école?

11 Ces fouilles ont permis d'approcher une partie seulement du village déserté connu sous le nom de «Ligny-la-Ville », mais elles apportent un certain nombre de faits nouveaux relatifs à cet habitat auparavant uniquement connu par les textes. Plusieurs grands bâtiments se dessinent à travers la présence de trous de poteaux de très grande taille et dénotent ce que l'on pourrait appeler «la construction en milieu funéraire " puisque plusieurs sarcophages ont été littéralement percés (et dans un cas le percement a détruit non seulement le couvercle mais également le fond de la cuve) pour l'installation de ces bâtiments. Cet intéressant cas de cohabitation "morts-vivants » pose la question des causes -foncières, juridiques, démographiques ?- à l'origine de cette inconfortable localisation.

12 Outre ces bâtiments, on peut également noter la présence d'un grand fossé et d'un grand fond de cabane de forme rectangulaire ${ }^{8}$, daté du XIV ${ }^{\mathrm{e}}$ siècle. Cet habitat vient enrichir le corpus assez faible finalement des villages désertés fouillés (pour le bas Moyen Âge et l'époque moderne) dont on peut regretter que l'étude, après une phase très active dans les années 1960-1970 , se soit étiolée pour aboutir à une quasi-atonie.

13 Cette fouille permet également de nourrir un dossier plus particulier: celui des agglomérations bipolaires dont il existe un certain nombre en Bourgogne et en Franche-Comté ${ }^{10}$. La dichotomie Ville / Châtel se retrouve à Ligny où les textes distinguent «Ligny-la-Ville» et «Ligny-le-Châtel ». L'étude de ce site est loin d'être terminée (une étude approfondie des archives reste à faire) mais il apparaît déjà 
comme un cas d'école où la création d'un château et d'une enceinte fortifiée à l'emplacement du bourg actuel va rapidement disqualifier "Ligny-la-Ville » dont les troubles liés à la guerre de Cent Ans vont sans doute précipiter le déclin et l'abandon définitif ${ }^{11}$.

Les perturbations, les destructions (par les labours en particulier), les dispersions occasionnées par les occupations successives (Âge du Bronze, Tène Finale, haut Moyen Âge, bas Moyen Âge) et de types différents (funéraire, habitat, agraire) ne facilitent pas la compréhension globale du site. Mais la confrontation des différentes disciplines et techniques utilisées sur ce chantier (étude pétrographique et typologique des sarcophages, étude typologique du mobilier et de l'évolution topo-chronologique de la nécropole, étude taphonomique, biologique et ADN des squelettes, études des sources d'archives, du parcellaire et du système viaire ${ }^{12}$, étude archéozoologique, etc.) laisse entrevoir un très grand potentiel qu'il appartiendra d'approfondir et de publier ${ }^{13}$.

\section{NOTES DE FIN}

1. L'étude de ce site étant encore en cours, seule la synthèse finale pourra valider les hypothèses émises dans cet article.

2. Nous remercions ici les services du Conseil Général de l'Yonne et le maire de LignyLe-Châtel qui ont permis le bon déroulement de cette opération.

3. Nous traitons ici seulement des vestiges de la période médiévale.

4. La première mention provient d'un précepte de l'empereur Louis le Débonnaire daté de 814 (cf. Gallia Christiana, t. V, col 129 ; M. Quantin, Cartulaire général de l'Yonne, Auxerre, t. I, 1854, p. 26).

5. Plutôt de la fin du vie siècle à la fin du vire siècle, mais la période d'utilisation des boucles en bronze couvre en amont tout le vie siècle.

6. Le terme de zone de contact n'est pas ici usurpé car la vallée du Serein est vraiment une zone de frontière, un point de rencontre ou convergent les limites de plusieurs civitates celtiques, diocèses et régions (Champagne, Bourgogne). D'un point de vue climatique, c'est également une zone de transition entre climat semi-continental et semi-océanique, une zone de contact entre « terres chaudes » calcaires propices aux vignobles de qualité et terres argileuses semblables à celles de la Champagne humide proche.

7. Pour la Champagne, notamment dans les nécropoles de la région de Mailly-le-Camp (Aube) (voir J.-P. Ravaux, La collection archéologique de Mme Perrin de La Boullaye, Châlonsen-Champagne, Musée de Châlons-en-Champagne, 1992) ; et dans l'Yonne, à Vaudonjon et à Saint-Martin-du-Tertre (informations communiquées par E. Poil).

8. Celui-ci a livré un abondant mobilier : céramique glaçurée orange (pichets), fragments de mortier, plaque-boucle en bronze, faune, etc...

9. Voir à ce sujet l'énorme publication riche d'informations et pluridisciplinaire proposant un ambitieux programme ayant abouti à une demi-douzaine de fouilles qui marqueront l'archéologie médiévale française (Dracy, Montaigu, Saint-Jean-le-Froid, Essertines) : Villages désertés et histoire économique (XI-XVIIIe s.), Paris, École Pratique des 
Hautes Études, Centre de Recherches Historiques, SEVPEN, 1965. Il serait peut-être intéressant, quarante ans après, de revenir sur cette problématique où l'archéologie n'est finalement intervenue que de façon marginale.

10. Berzé-la-Ville / Berzé-le-Châtel, Pagny-la-Ville / Pagny-le-Châtel, Norges-la-Ville / Norges-le-Bas, Montmirey-la-Ville / Montmirey-le-Château.

11. Ces troubles ont fortement affecté la région auxerroise. Le secteur fouillé en 2006 donne comme date d'abandon le xive siècle.

12. Notamment à partir de photos aériennes : plusieurs dizaines de vues (verticales et obliques) du site ont été réalisées à partir d'un dirigeable et d'un avion durant la fouille. 13. Une première série d'actions entreprises auprès de la population locale (une journée portes ouvertes, l'installation de panneaux et le dépôt de sarcophages dans l'église de Ligny, l'édition de plaquettes, la présentation du site dans les écoles) a déjà permis de faire connaître les premiers résultats de cette fouille

\section{INDEX}

Mots-clés : village déserté, nécropole mérovingienne

Index géographique : France/Ligny-le-Châtel 